\title{
Influential Factors of Successful Hepatitis C Treatment in Elderly Patients
}

Jennifer Andres, PharmD, BCPS; Mandee Noval, PharmD; Christine Mauriello, PharmD; Derek Peiffer, PharmD; Huaqing Zhao, PhD

Temple University School of Pharmacy

\begin{abstract}
Background: Chronic Hepatitis C virus (HCV) is an infection associated with an increased risk of cirrhosis, hepatocellular carcinoma (HCC), and morbidity and mortality. Treating HCV poses challenges in the elderly population due to the lack of evidence and complexity of patients.

Objective: This study aims to evaluate factors that influence HCV treatment success in elderly patients, especially those over age of 70, such as pill burden and comorbidities, in addition to drug interactions and adverse effects.

Methods: This was a retrospective chart review of patients treated at our urban academic institution from 2014-2016.

Results: Sixty-two patients over the age of 70 were included in this study. The sustained virologic response rate 12 weeks after the completion of treatment (SVR12) was 79\%. In a multi-variate analysis, cirrhosis, age closer to 70, and longer duration of treatment were statistically significantly more likely to lead to treatment failure. Though not statistically significant, other factors that may negatively influence achievement of SVR12 were cognitive impairment, cardiovascular disease, multi-tablet HCV regimen, time to initiation of HCV treatment > 90 days, and prior treatment experience. Pill burden of other prescribed medications did not impact SVR12. Adverse events and drug interactions were common in the population.

Conclusions: Overall SVR12 rate in the elderly population was lower than that reported in the literature. Factors associated with lower treatment success, especially cirrhosis, should be considered when treating an elderly population. Further data is needed on the impact of other factors on SVR12 attainment in an elderly patient population.
\end{abstract}

Keywords: Hepatitis C, Elderly, Polypharmacy, Drug Interactions, Adverse Events

\section{Introduction}

Chronic Hepatitis C virus (HCV) is an infection associated with an increased risk of cirrhosis, hepatocellular carcinoma (HCC), and morbidity and mortality. ${ }^{1} \mathrm{HCV}$ can also cause extrahepatic manifestations such as diabetes, renal impairment, cognitive impairment, and cardiovascular disease, among others. ${ }^{2}$ It is estimated that almost 4 million people are affected by HCV in the United States (US). Although HCV can affect all age groups, one of the most impacted groups is estimated to be in those born between the years of 1945-1965.,4 Patients in this age cohort are beginning to turn 70 years of age and soon all members of this cohort will exceed 70 years of age.

It is imperative to understand how age affects cirrhosis due to the aging population of patients with HCV. Cirrhosis is an advanced degree of fibrosis with hepatic vasculature alterations that lead to portal hypertension and end-stage liver disease. ${ }^{5.6}$ Studies have demonstrated that increased duration of HCV infection are risk factors for progression to worsening fibrosis stages and cirrhosis. ${ }^{7,8}$ Cirrhosis most commonly affects patients 60 to 80 years of age. ${ }^{9}$ A retrospective cohort of Veterans Affairs (VA) patients demonstrated that the age of patients with cirrhosis has increased from a mean 57.3 years

Corresponding author: Jennifer Andres, PharmD, BCPS

Temple University School of Pharmacy

Email: jennifer.andres@temple.edu
(SD 10.5) in 2001 to 62.4 (SD 8.4) in 2013 and HCV was seen in an increased proportion of patients. ${ }^{10}$ Achieving clinical cure or sustained virologic response (SVR) 12 weeks (SVR12) after treatment completion decreases the risk of cirrhosis development. In cirrhotic patients, achieving SVR12 can decrease the likelihood of decompensation and lower the risk of $\mathrm{HCC}^{3}$

Prior to 2011, HCV treatment was interferon (IFN) -based, leading to increased adverse effects (AEs) and poor patient outcomes, especially in an elderly population. ${ }^{11}$ Many elderly patients were not treated due to comorbidities affecting eligibility and AE profiles. Since then, highly effective oral direct acting antivirals (DAAs) have been approved by the Food and Drug Administration and have significantly improved patient outcomes with SVR12 rates exceeding 90\%, however few elderly patients were included in these studies. ${ }^{1,3,12,13}$ The high efficacy and decreased pill burden of DAAs was accompanied by high costs of therapy and delays in access. A retrospective cohort study including 9025 patients from 45 states found that $35.5 \%$ were denied treatment, with the largest number coming from commercial insurance companies. ${ }^{14}$ Often, patients were prioritized for treatment from insurance companies based on presence of more severe hepatic dysfunction and HIV coinfection. When elderly patients have been evaluated in realworld studies, SVR12 rates were comparable to the general population. ${ }^{15-17}$ Despite similar SVR12 rates, elderly patients remain difficult to treat due to increasing presence of comorbid disease states, which may be worsened by HCV infection, and 
higher pill burden. Both factors may increase the likelihood of drug interactions (DIs) and AEs.

Over $87 \%$ of the US population between the ages of 62 and 85 takes at least one medication daily according to a 2011 survey and $35 \%$ take more than 5 medications daily. ${ }^{18}$ Adherence to medication is a contributor to achieving optimal outcomes. Pill burden, or number of pills a patient must take per day, may impact adherence and thus, HCV treatment success. Higher pill burden has demonstrated negative effects on adherence and outcomes in diseases such as hypertension and human immunodeficiency virus (HIV) and should be considered when selecting therapy in the elderly population. ${ }^{19-22}$ Data from the Accurate Dosing in Hepatitis C: Examining the RibaPak Experience (ADHERE) registry assessed how traditional RBV and RibaPak (RBP) affected HCV treatment adherence, since RBP is a twice daily regimen with lower pill burden. ${ }^{23}$ Patients using RBP were more adherent to therapy, defined as taking $\geq 80 \%$ of doses, at both 12 weeks and 24 weeks compared to those using RBV ( $p=0.02$ and $p<0.05$, respectively), but no clinical outcomes were assessed. ${ }^{23}$ No studies on the effect of pill burden on adherence and clinical outcomes has been published in the DAA era.

The prevalence of DIs in patients taking DAAs is relatively high, due to their effects on multiple transporter systems and altered drug metabolism. Elderly patients may be at a greater risk for DIs and AEs, due to a larger number of concomitant medications for multiple comorbidities. ${ }^{25,26}$ A small retrospective study from Germany described the DIs and adverse effects faced by elderly patients receiving DAAs. ${ }^{24}$ The proportion of predicted clinically significant DIs was significantly higher in patients greater than 65 years of age, ( $54 \%$ vs. $28 \% ; P<0.0001$ ) indicating that medication use should be evaluated, but SVR12 was high in patients over age $65 .{ }^{24} \mathrm{~A}$ study with 221 patients on DAAs with or without RBV showed that participants of older age, defined as greater than 75 years, were more likely to experience a more serious (grade 3-4) AE. ${ }^{16}$

Our institution is a large academic medical center in an urban environment serving a large number of patients with multiple comorbidities. Upon the decision to treat HCV by a provider, a clinical pharmacist meets with the patient to initiate and manage DAA treatment. Once a DAA is started, the patient is followed by a clinical pharmacist, a nurse, and a provider. This study aims to evaluate other factors, such as pill burden and comorbidities, that impact HCV treatment outcomes in elderly patients, including DDIs and AEs.

\section{Methods}

This was a single-center retrospective evaluation of HCV monoinfected patients who were at least 70 years old, or "elderly" patients, at the time of $\mathrm{HCV}$ therapy initiation at our institution. EPIC (Epic Systems Corporation, Verona, Wisconsin) medical records were evaluated for all patients that began DAA therapy between June 2014 and September 2016 in the Hepatology and Gastroenterology departments at our institution. Treatment regimens and duration were determined based on standard of care guidelines and insurance coverage at the time of treatment. $^{3}$

The primary outcome was the achievement of SVR12, defined by an undetectable HCV ribonucleic acid (RNA) twelve weeks after completion of DAAs. Secondary outcomes included SVR12 by regimen, comorbidity, treatment experience, pill burden, and time to treatment initiation. The number and type of DIs and reported AEs were also evaluated. Comorbidites of interest, defined by the problem list or laboratory findings in EPIC, included cirrhosis (Fibrosis stage of F4 and progress note documentation), cognitive impairment (dementia or cognitive impairment), renal dysfunction (Estimated Glomerular Filtration Rate (EGFR) $<60 \mathrm{~mL} / \mathrm{min}$ ), diabetes (HgbA1C $>6.5 \%$ ), and cardiovascular disease. Medication and pill burden was determined by the counting the number of oral medications and the tablets and/or capsules (referred to as pills) that a patient was taking on the start date of DAAs as documented in EPIC. Medications taken as needed were assessed by medication count and pill burden. A medication dosed weekly was counted as one pill. Time to treatment initiation was calculated as the time between the initial decision to treat per progress notes and the date the patient began DAAs. Clinic visit notes were reviewed to determine if AEs occurred and if DIs existed.

All patients over age 70 treated for HCV within the defined period were included. No patients were excluded. Categorical variables were expressed as percentages, and continuous variables were expressed as medians with interquartile ranges. Pearson chi-square test was performed for categorical variables and a two-sample t-test for continuous variables. A multivariable logistic regression model evaluated variables that predicted SVR based on a $p$-value $<0.1$ with univariate analysis. Spearman test was used to determine correlation between variables prior to performing multivariable logistic regression. AORs with $95 \% \mathrm{Cls}$ are reported for the final logistic regression models. All statistical analyses were performed using Stata release 14 (StataCorp, College Station, Texas). An a priori significance level of 0.05 was used.

\section{Results}

Sixty-two patients were included in the study and received HCV treatment. Table 1 provides pertinent demographic information. Among the participants, the mean [SD] age was 73 [2.88] years and most patients were female and African American. Over $90 \%$ of patients had genotype $1 \mathrm{a}$ or $1 \mathrm{~b}$ and were treatment naive. Cirrhosis was evident in $58 \%$ of patients. Other comorbidities present included diabetes, cardiovascular disease, cognitive impairment, and renal impairment evidenced by an estimated eGFR $<60 \mathrm{ml} / \mathrm{min}$. 
The primary outcome of SVR12 achievement was assessed in 61 patients (1 patient lost to follow-up). Of 61 patients, 49 (79\%) achieved SVR12 overall. The average age of patients who achieved SVR12 was older than the treatment failures ( 74.04 vs 70.67, $\mathrm{p}=0.003$ ). Table 2 summarizes factors evaluated as influencers of SVR12 attainment based on the univariate analysis. SVR12 was achieved in statistically significantly more non-cirrhotic patients than with cirrhosis $(p=0.001)$. The presence of diabetes, cardiovascular disease, and cognitive impairment was associated with a lower rate of SVR12, however only the presence of cognitive impairment $(p=0.049)$ was statistically significantly associated with treatment failure. Twenty-two patients had more than one comorbid condition. Presence of multiple comorbidities reduced SVR12 rate; treatment failures had an average of 2.16 conditions, while successful treatment had an average of 1.32 condition $(p=0.02)$. As number of comorbidities increased, SVR12 was $73 \%$ in those with 2 or more comorbidities, but decreased to $50 \%$ in the 4 patients with 4 or more comorbidities achieved SVR12.

Individual SVR12 rates varied among agents: LDV/SOF +/- RBV $82 \%, \mathrm{SMV} / \mathrm{SOF} 40 \%$, and $100 \%$ for SOF/RBV, EBR/GZR, and other regimens (PrOD + RBV) and SOF + daclatasvir + RBV. Of the 12 patients without SVR12 achievement, five received LDV/SOF for 12 weeks, three LDV/SOF for 24 weeks, one LDV/SOF + RBV for 24 weeks, one SIM/SOF for 12 weeks, and two SMF/SOV for 24 weeks. Patients with genotype 1 had an SVR12 of $78.5 \%$, while patients with mixed genotypes or genotype 2 had an SVR12 of $100 \%$. There was no statistical difference in SVR12 achievement by genotype. Overall, SVR12 rates were greatest in those treated for shorter durations compared to 24 weeks ( 8 weeks $100 \%$; 12 weeks $83.78 \%$; 16 weeks 100\%; 24 weeks 50\%). Duration of treatment significantly impacted SVR12. The 12 treatment failures were treated for a mean of 18 weeks compared to the 49 successfully treated patients taking DAAs for a mean of 12.8 weeks $(p=0.002)$. SVR12 was achieved in more patients on a singletablet daily HCV regimen than on a multi-tablet HCV regimen. The 22 treatment experienced patients achieved SVR12 at a lower rate than treatment naive patients.

The average number of concomitant medications that patients were taking daily was approximately 8 , ranging from 1 to 29 medications/day. Due to several medications requiring multiple administrations daily, the average daily pill burden was approximately 9 tablets/day with a range of 1 to 33 tablets/day. Several patients also reported taking PRN medications, with an average of 2 tablets/day and a range of 0-14 tablets/day. Of patients taking 9 or more medications daily, $82 \%$ achieved SVR12 ( $n=27)$. In contrast, those taking less than 9 tablets daily reported similar results with approximately $79 \% \quad(n=22)$ attaining SVR12.

The average time to treatment initiation was approximately 97 days. A delay in treatment initiation was reported in $80 \%(n=50)$ of patients, with the most commonly reported reason for delay being insurance authorization in $46 \%(n=29)$ of cases. Other reasons noted for delayed treatment included pending lab results $(n=10)$, communication difficulties with patients $(n=7)$, and other health concerns $(n=8)$ including altered mental status, emergency room visits, or awaiting other procedures. Several patients had multiple reasons for treatment delay (i.e. pending insurance approval, requiring lab results) and 19\% $(n=12)$ did not have reasons listed regarding treatment delays. In the 23 patients waiting more than 90 days to begin treatment, SVR12 was lower than in the 38 patients waiting less than 90 days to begin treatment.

The multivariate analysis evaluated age at treatment start, treatment experience, number of comorbidities, duration of treatment, and presence of cognitive impairment. Due to the relation between number of comorbidities and treatment duration, two multivariate models were conducted. Older age at treatment start (AOR 1.88; 95\% $\mathrm{Cl} 1.075-3.303 ; \mathrm{p}=0.027$ ) and shorter duration of HCV treatment (AOR 0.87 ; $95 \% \mathrm{Cl} 0.763$ $0.989 ; p=0.034$ ) were found to significantly predict treatment success in one multivariate model. In the second model, older age persisted as a treatment success predictor (AOR 2.11; 95\% $\mathrm{Cl} 1.16-3.85 ; \mathrm{p}=0.014)$ as did a lower number of comorbidities (AOR 0.52; 95\% Cl 0.262-1.042; $\mathrm{p}=0.66$ ).

Potential DIs were reported in $45 \%(n=28)$ of patients, resulting in discontinuation or dose adjustment of non-HCV medications prior to DAA initiation. Overall, 15 medications were discontinued and 13 medications required adjustments in dose or time of administration. The most common medications implicated in DIs included acid-reducing agents with at least 8 patients requiring discontinuation or alteration in administration time. Other medications that were commonly implicated include amlodipine $(n=3)$ and statins (lovastatin, rosuvastatin, simvastatin) ( $n=3)$. Of note, several patients experienced fluctuations in drug levels requiring dose adjustments, including both tacrolimus $(n=1)$ and warfarin $(n=1)$. Several patients had other medication changes during DAA treatment, though unrelated to DIs. During treatment, 32 participants (52\%) experienced one or more AEs. Fatigue (24\%), headache $(8 \%)$, appetite changes $(8 \%)$, and $\mathrm{Gl}$ abnormalities (8\%) were most commonly reported. Less common AEs included myalgias (5\%), hypokalemia (1.5\%), and edema (1.5\%). In patients taking RBV, anemia was not reported, however nonspecific AEs such as fatigue were reported. Several patients reported incidence of multiple AEs listed, however no participants discontinued treatment due to AEs.

\section{Discussion}

The average age of this patient population was 73 years old, correlating with the baby boomer population recommended for HCV screening. Interestingly, younger patients in our cohort achieved SVR12 less than older patients in our cohort, though the mean age difference was small (3.3 years). As can be 
expected with an older patient population, many presented with comorbidities. When patients presented with multiple comorbidities, their chance of achieving SVR12 decreased. Cirrhosis was found in $53 \%$ of patients, likely as a result of a long duration of chronic HCV infection. Presence of cirrhosis was found to be the comorbidity that impacted SVR12 rate most. DAAs were shown to have lower treatment success in a real world study of 15,884 VA patients with cirrhosis [ $86.8 \%(95 \% \mathrm{Cl}$ 85.8-87.7)] or decompensated cirrhosis [82.6\% (95\% Cl 80.584.6)] compared to those without cirrhosis $[92.3 \%(95 \% \mathrm{Cl}$ 91.8-92.8)], regardless of age. ${ }^{27}$ Other comorbidities found to have a negative effect on SVR12 in our study included diabetes, cardiovascular disease, and cognitive impairment. While cirrhosis has been documented to have an impact on treatment response, this is the first data evaluating the impact of multiple comorbidities, including diabetes, cardiovascular disease, and cognitive impairment on treatment outcomes. Due to the small number of patients in this evaluation, larger studies should evaluate the impact of the comorbidities on SVR12.

SVR12 was attained more frequently in patients receiving single tablet HCV regimens compared to multiple tablet regimens. This may be attributed to a lower pill burden, leading to an increased chance of adherence. Patients on multiple tablet DAAs may have been more likely to have cirrhosis, but the addition of RBV should have helped improve SVR12 rates in this population. Patients with cirrhosis were also more likely to be on a treatment duration longer than 12 weeks. SIM/SOF had the lowest SVR12 rate. Four of the patients had cirrhosis and three were treatment experienced; both can lead to lower SVR12 achievement. ${ }^{3}$ Although predicted that overall pill burden would have an effect on clinical cure, SVR12 rates in patients taking $>9$ pills/day were similar to those taking $<9$ pills/day. Adherence to medications was not systematically measured, thus it is difficult to determine the impact of pill burden. Patients may have been on background medications for any period of time before HCV treatment and been adherent, so pill burden may not have impacted adherence and treatment success in this patient population. Long delays in treatment, such as waiting to treat until patients have attained a higher fibrosis stage, may lead to decreased incidence of SVR12 attainment and a higher mortality risk. ${ }^{28,29}$ Specific shortterm delays once decision is made to treat have not yet been evaluated. Patients experiencing a treatment delay of $>90$ days resulted in a $17 \%$ decrease in SVR12 achievement in this evaluation. Thus, it is vital to initiate treatment promptly to prevent treatment failure.

Dls were reported in $45 \%$ of patients and led to pharmacotherapy changes. Many DAAs have known Dls, which require dosing adjustments and/or increased monitoring of other therapies. ${ }^{30}$ The high rate of Dls was expected and is generally consistent with those discussed in the literature. ${ }^{24}$ Over half of the patients experienced AEs, but no patients discontinued treatment, which may infer that AEs were minimal and tolerated by patients. Patients in this study showed similar rates to those previously reported. ${ }^{16}$

This analysis has several limitations due to the retrospective design. Some data points were not available for every patient. Only one patient did not receive follow up care at our institution and was not included in the results. Follow up rates are generally lower in real world analyses; this may indicate that elderly patients are more likely to be engaged in care by returning for follow up. In comparison to a clinical trial, this analysis has a smaller size, lacks a comparator group, and was conducted at a single center, making it hard to determine if these results are similar in other institutions or among our entire population. A larger analysis comparing our elderly population to other centers and comparing our elderly population to our nonelderly population would help to assess this. There is debate over the definition of elderly by a person's chronological age, with a starting age ranging from $60-75 .{ }^{31} \mathrm{By}$ choosing patients over 70 years, we better captured the aging population with comorbidities, however we decreased the sample size. Additionally, this analysis utilized descriptive statistics with some use of statistical tests. A post-hoc multivariate analysis was conducted, however applicability is limited due to the small number of patients and treatment failures in this analysis. A more robust, multi-variate analysis with more patients will be necessary to determine true impact of variables on treatment. While the DAAs used in this evaluation were highly effective, several new regimens have been approved since 2016, limiting the use of some regimens that were included in this analysis. The use of PrOD, SOF/SIM, SOF/RBV are no longer first line treatments due to the lower efficacy rates in some populations such as cirrhotics, and negative characteristics such as higher pill burden and drug interactions. While SIM/SOF had the lowest rate of SVR12 achievement, the use of other regimens did not correspond to lower SVR12 rates. The majority of patients received DAA regimens that are recommended by current guidelines, indicating that these findings could be replicated with current treatments. Though not statistically significant, the use of a multiple tablet DAAs negatively impacted SVR12 results in our population. Inclusion of newer regimens in our population would have helped determine if this is similar with recently approved regimens containing more than one tablet daily.

Our institution is located in an urban setting, and thus this analysis includes more Hispanic patients and African American patients than typical clinical trials. More females were treated in our elderly cohort than typical clinical trials. Patients primarily presented with genotype $1 \mathrm{a}$ and $1 \mathrm{~b}$, which are the most common genotypes found in the US, increasing the generalizability of our results. Based on these findings, clinicians should be cognizant of the considerations needed for an elderly population. Clinical pharmacists should assess for drug-drug interactions and encourage medication adherence and treatment of patients as soon as HCV is discovered. 


\section{Conclusion}

The SVR12 in patients over the age of 70 at our institution was lower than that of the general population. Most treatment failures occurred in elderly patients with cirrhosis or other comorbidities such as diabetes, cardiovascular disease, or cognitive impairment, likely a result of patients living with HCV for longer. Higher overall pill burden did not impact attainment of SVR12, however DAAs with more than one tablet daily decreased SVR12 rates, which could be a result of cirrhotic and/or treatment experienced patients. Patients with higher pill burden or comorbidities are also at an increased risk of DDIs and AEs so additional precautions and monitoring should be taken. Further data is needed to confirm the impact of these and other factors on SVR12 attainment in an elderly patient population.

\section{Conflict of Interest: None \\ Funding: None}

\section{References}

1. Hepatitis C FAQs for Health Professionals. Available at: https://www.cdc.gov/hepatitis/hcv/hcvfaq.htm. Last updated 30 April 2018. Accessed 7 June 2018.

2. Cacoub P. Extrahepatic manifestations of chronic hepatitis C virus infection. Ther Adv Infect Dis. 2016; 3(1): 3-14.

3. AASLD-IDSA. Recommendations for testing, managing, and treating hepatitis $\mathrm{C}$. http://www.hcvguidelines.org. Updated 24 May 2018. Accessed 6 June 2018.

4. Smith B, Morgan R, Beckett G, et al. Hepatitis C Virus Testing of Persons Born During 1945-1965: Recommendations from Center for Disease Control and Prevention. Ann Intern Med. 2012; 157(11):817822.

5. Schuppan D and Afdhal NH. Liver cirrhosis. Lancet. 2008; 371:838-851.

6. Poynard T, Bedossa P, and Opolon P. Natural history of liver fibrosis progression in patients with chronic hepatitis C. Lancet. 1997; 349:825-832.

7. Poynard T, Ratziu V, Charlotte F, Goodman Z, McHutchison J, and Albrecht J. Rates and risk factors of liver fibrosis progression in patients with chronic hepatitis C. Journal of Hepatology. 2001; 34(5): 730739.

8. Becker U, Deis A, Sorensen TIA, Gronbaek M, BorchJohnsen, et al. Prediction of risk of liver disease by alcohol intake, sex, and age: a prospective population study. Hepatology, 1996; 23:1025-1029.

9. Mindikoglu AL, Miller RR. Hepatitis C in the Elderly: Epidemiology, Natural History, and Treatment. Clinical Gastroenterology and Hepatology. 2009; 7(2):128-134.
10. Rheem J, Sundaram V, and Saab S. Antiviral Therapy in Elderly Patients With Hepatitis C Virus Infection. Gastroenterol Hepatol (N Y). 2015; 11(5): 294-346.

11. Conti F, Brillanti S, Buonfiglioli F. Safety and efficacy of direct-acting antivirals for the treatment of chronic hepatitis $C$ in a real-world population aged 65 years and older. J Viral Hepat. 2017;24(6):454-463.

12. Ishida K, Shimakami T, Kaneko S. The use of directacting antivirals in the treatment of elderly patients with hepatitis C Virus infection. Nihon Ronen Igakkai Zasshi. 2017;54(3):375-380.

13. Lam, B et.al. The changing landscape of hepatitis $c$ virus therapy: focus on interferon-free treatment. 2015; 8(5): 298-312

14. Gowda C, Lott S, Grigorian M, Carbonari DM, Saine $\mathrm{ME}$, Trooskin $\mathrm{S}$, et al. Absolute Insurer Denial of Direct-Acting Antiviral Therapy for Hepatitis C: A National Specialty Pharmacy Cohort Study. Open Forum Infect Dis. 2018;5(6):ofy076.

15. Snyder HS, Ali B, Gonzalez HC, Nair S, Satapathy SK. Efficacy and Safety of Sofosbuvir-Based Direct Acting Antivirals for Hepatitis $C$ in Septuagenarians and Octogenarians. J Clin Exp Hepatol. 2017;7(2):93-96.

16. Fabrizio C, Saracino A, Scudeller L, et al. The elderly and direct antiviral agents: Constraint or challenge? Digestive and Liver Disease. 2017; 49(9):1036-1042.

17. Rodríguez-Osorio I, Cid P, Morano L. Real life experience with direct-acting antivirals agents against hepatitis C infection in elderly patients. J Clin Virol. 2017;88:58-61.

18. Qato DM, Wilder J, Schumm LP, Gillet V, Alexander GC. Changes in Prescription and Over-the-Counter Medication and Dietary Supplement Use Among Older Adults in the United States, 2005 vs 2011. JAMA Intern Med. 2016;176(4):473-82.

19. Carter BL, Bergus GR, Dawson JD. et al. A cluster randomized trial to evaluate physician/pharmacist collaboration to improve blood pressure control. J Clin Hyperten. 2008; 10: 260-271.

20. Libby, AM, Fish, DN, Hosokawa, PW. Patient-level medication regimen complexity across populations with chronic disease. Clin Ther. 2013; 35:385-398.e1.

21. Mohd Salleh NA, Richardson L, Kerr T, et al. A Longitudinal Analysis of Daily Pill Burden and Likelihood of Optimal Adherence to Antiretroviral Therapy Among People Living With HIV Who Use Drugs. Journal of Addiction Medicine. 2018;1-7.

22. Sutton SS, Magagnoli J, and Hardin JW. Impact of Pill Burden on Adherence, Risk of Hospitalization, and Viral Suppression in Patients with HIV Infection and AIDS Receiving Antiretroviral Therapy. Pharmacotherapy. 2016;36(4): 385-401. 
23. Alam I, Stainbrook T, Cecil B, and Kistler KD. Enhanced adherence to HCV therapy with higher dose ribavirin formulation: Final analyses from the ADHERE registry. Aliment Pharmacol Ther. 2010; 32(4): 535-542.

24. Vermehren J, Peiffer $\mathrm{KH}$, Welsch C, et al. The efficacy and safety of direct acting antiviral treatment and clinical significance of drug-drug interactions in elderly patients with chronic hepatitis $C$ virus infection. Aliment Pharmacol Ther. 2016; 44: 856865.

25. Brahma DK, Wahlang JB, Marak MD, Sangma MCH. Adverse drug reactions in the elderly. Journal of Pharmacology \& Pharmacotherapeutics. 2013;91-94

26. Beijer $\mathrm{H}$, de Blaey $\mathrm{C}$. Hospitalizations caused by adverse drug reactions (ADR): a meta-analysis of observational studies. Pharm World Sci. 2002; 24: 4654.

27. Su F, Beste L, Green P, Berry K, and loannou G. Directacting antivirals are effective for chronic hepatitis $C$ treatment in elderly patients: A real-world study of 17487 patients. European Journal of Gastroenterology \& Hepatology. 2017; 29(6): 686693.
28. Jezequel C, Bardou-Jacquet E, Desille $Y$, et al. Survival of patients infected by chronic hepatitis $C$ and FOF1 fibrosis at baseline after a 15 year follow-up. 50th Annual Meeting of the European Association for the Study of the Liver (EASL). 2015:S589.

29. McCombs JS, Tonnu-MiHara I, Matsuda T, McGinnis J, Fox $S$. Can hepatitis $C$ treatment be safely delayed? Evidence from the Veterans Administration Healthcare System. 50th Annual Meeting of the European Association for the Study of the Liver. 2015;S191.

30. Burger D, Back $P$, Buggisch $M$, et al. Clinical management of drug-drug interactions in $\mathrm{HCV}$ therapy: Challenges and solutions. Journal of Hepatology. 2012; 58(4):792-800.

31. Singh S. Defining 'elderly' in clinical practice guidelines for pharmacotherapy. Pharm Pract. 2014; 12(4): 489. 
Table 1: Characteristics of Elderly Population $>70$ Years of Age

\begin{tabular}{|c|c|}
\hline Variable & Value (Total $n=62$ ) \\
\hline Age (range), years & $73 \pm 2.88(70-86)$ \\
\hline Female Gender & $61 \%(n=38)$ \\
\hline \multicolumn{2}{|l|}{ Race } \\
\hline African American & $74 \%(n=46)$ \\
\hline Caucasian & $15 \%(n=9)$ \\
\hline Hispanic & $11 \%(n=7)$ \\
\hline \multicolumn{2}{|l|}{ Comorbidities } \\
\hline Cirrhosis & $58 \%(n=36)$ \\
\hline Diabetes & $31 \%(n=19)$ \\
\hline Cardiovascular Disease & $21 \%(n=13)$ \\
\hline Cognitive Impairment & $10 \%(n=6)$ \\
\hline eGFR $<60 \mathrm{ml} / \mathrm{min}$ & $31 \%(n=19)$ \\
\hline Treatment Naive & $65 \%(n=40)$ \\
\hline \multicolumn{2}{|l|}{ Genotype } \\
\hline $1 a$ & $51 \%(n=32)$ \\
\hline $1 b$ & $40 \%(n=25)$ \\
\hline 2 & $5 \%(n=3)$ \\
\hline $1 a / 1 b$ & $2 \%(n=1)$ \\
\hline $1 a / 3$ & $2 \%(n=1)$ \\
\hline \multicolumn{2}{|l|}{ DAA Regimen } \\
\hline \multicolumn{2}{|l|}{ Ledipasvir/Sofosbuvir (LDV/SOF) } \\
\hline 8 weeks & $17 \%(n=11)$ \\
\hline 12 weeks & $45 \%(n=28)$ \\
\hline 24 weeks & $14 \%(n=9)$ \\
\hline \multicolumn{2}{|c|}{ Ledipasvir/Sofosbuvir + Ribavirin (LDV/SOF + RIB) } \\
\hline 12 weeks & $n=1$ \\
\hline 24 weeks & $n=1$ \\
\hline
\end{tabular}




\begin{tabular}{|c|c|}
\hline \multicolumn{2}{|c|}{ Simeprevir + Sofosbuvir (SIM/SOF) } \\
\hline 212 weeks & $4 \%(n=3)$ \\
\hline \multicolumn{2}{|c|}{$3 \%(n=2)$} \\
\hline 12 weeks & $n=1$ \\
\hline 16 weeks & $3 \%(n=2)$ \\
\hline Elbasvir/Grazoprevir (EBR/GZR) & $3 \%(n=2)$ \\
\hline 12 weeks & $3 \%(n=2)$ \\
\hline Other $^{a}$ & \\
\hline
\end{tabular}

${ }^{a}$ Other regimens include PrOD + RBV and SOF/daclatasvir + RBV 
Table 2: Summary of Contributing Factors on SVR12 Attainment ${ }^{\mathrm{a}}$

\begin{tabular}{|c|c|c|c|}
\hline Contributing Factor & $\begin{array}{l}\text { Attained SVR12 } \\
\text { With Contributing } \\
\text { Factor }\end{array}$ & $\begin{array}{c}\text { Attained SVR12 } \\
\text { Without } \\
\text { Contributing Factor }\end{array}$ & $P$ value \\
\hline \multicolumn{4}{|l|}{ Comorbidity } \\
\hline Cirrhosis & $67 \%$ & $100 \%$ & 0.001 \\
\hline Diabetes & $68 \%$ & $83.7 \%$ & 0.303 \\
\hline Cardiovascular Disease & $77 \%$ & $81.25 \%$ & 0.728 \\
\hline Cognitive Impairment & $50 \%$ & $83.63 \%$ & 0.049 \\
\hline \multirow[t]{2}{*}{ eGFR $<60 \mathrm{ml} / \mathrm{min}$} & $84 \%$ & $80.95 \%$ & 0.608 \\
\hline & \multicolumn{2}{|c|}{ SVR12 Attainment by Contributing Factor } & \\
\hline \multirow[t]{2}{*}{ HCV Regimen } & $\begin{array}{l}\text { Single Tablet } \\
\text { Regimen }\end{array}$ & $\begin{array}{l}\text { Multi Tablet } \\
\text { Regimen }\end{array}$ & \\
\hline & $83.6 \%$ & $66.7 \%$ & 0.184 \\
\hline \multirow[t]{2}{*}{ Treatment History } & Treatment Naive & $\begin{array}{l}\text { Treatment } \\
\text { Experienced }\end{array}$ & \\
\hline & $87 \%$ & $68 \%$ & 0.073 \\
\hline \multirow[t]{2}{*}{ Pill Burden } & $\geq 9$ tablets/day & $<9$ tablets/day & \\
\hline & $82 \%$ & $79 \%$ & 0.655 \\
\hline \multirow[t]{2}{*}{ Time to HCV Treatment } & $<90$ days & $\geq 90$ days & \\
\hline & $86.84 \%$ & $69.56 \%$ & 0.100 \\
\hline
\end{tabular}

${ }^{\text {a }}$ Factors evaluated using univariate analysis 